\title{
Disruption of the Splicing Enhancer Sequence within Exon 27 of the Dystrophin Gene by a Nonsense Mutation Induces Partial Skipping of the Exon and Is Responsible for Becker Muscular Dystrophy
}

\author{
Nobuyuki Shiga, Yasuhiro Takeshima, ${ }^{*}$ Hiroshi Sakamoto, ${ }^{\S}$ Kunio Inoue,, Yoshiyuki Yokota, ${ }^{\mathbb{\complement}}$ Mitsuhiro Yokoyama, ${ }^{\ddagger}$ \\ and Masafumi Matsuo \\ Division of Genetics, International Center for Medical Research, *Department of Pediatrics, and ${ }^{\ddagger}$ First Department of Internal Medicine, \\ Kobe University School of Medicine, Kobe 650, Japan; ${ }^{\circledR}$ Department of Biology, Kobe University Faculty of Science, Kobe 657, Japan; \\ "Department of Biological Science, Nara Institute of Science and Technology, Nara 630-01, Japan; and "Faculty of Health Science, Kobe \\ University School of Medicine, Kobe 654-01, Japan
}

\begin{abstract}
The mechanism of exon skipping induced by nonsense mutations has not been well elucidated. We now report results of in vitro splicing studies which disclosed that a particular example of exon skipping is due to disruption of a splicing enhancer sequence located within the exon. A nonsense mutation (E1211X) due to a $\mathrm{G}$ to $\mathrm{T}$ transversion at the 28th nucleotide of exon 27 (G3839T) was identified in the dystrophin gene of a Japanese Becker muscular dystrophy case. Partial skipping of the exon resulted in the production of truncated dystrophin mRNA, although the consensus sequences for splicing at both ends of exon 27 were unaltered.

To determine how E1211X induced exon 27 skipping, the splicing enhancer activity of purine-rich region within exon 27 was examined in an in vitro splicing system using chimeric doublesex gene pre-mRNA. The mutant sequence containing G3839T abolished splicing enhancer activity of the wild-type purine-rich sequence for the upstream intron in this chimeric pre-mRNA. An artificial polypurine oligonucleotide mimicking the purine-rich sequence of exon 27 also showed enhancer activity that was suppressed by the introduction of a $\mathrm{T}$ nucleotide. Furthermore, the splicing enhancer activity was more markedly inhibited when a nonsense codon was created by the inserted $T$ residue. This is the first evidence that partial skipping of an exon harboring a nonsense mutation is due to disruption of a splicing enhancer sequence. (J. Clin. Invest. 1997. 100:2204-2210.) Key words: dilated cardiomyopathy $\cdot$ reading frame $\bullet$ in vitro splicing $\bullet$ pre-mRNA $\cdot$ polypurine
\end{abstract}

\section{Introduction}

Pre-mRNA splicing demands remarkable accuracy. The cisacting elements implicated in splicing are the $5^{\prime}$ and $3^{\prime}$ splice site sequences, branch point sequences, and their locations (1).

Address correspondence to Masafumi Matsuo, M.D., Ph.D., International Center for Medical Research, Kobe University School of Medicine, 7-5-1 Kusunokicho, Chuo, Kobe 650, Japan. Phone: 81-78-3417451; FAX: 81-78-362-6064; E-mail: matsuo@kobe-u.ac.jp

Received for publication 11 May 1997 and accepted in revised form 27 August 1997.

J. Clin. Invest.

(C) The American Society for Clinical Investigation, Inc. 0021-9738/97/11/2204/07 \$2.00

Volume 100, Number 9, November 1997, 2204-2210

http://www.jci.org
Significantly, these elements are necessary but not sufficient for intron removal. Several lines of evidence indicate that a purine-rich region within the exon sequence, the splicing enhancer sequence $(\mathrm{SES})^{1}$ or exon recognition sequence, operates as a cis-element to enhance splicing of the upstream intron (2-4). Recently, members of the SR family of essential splicing factors, characterized by alternating serine and arginine residues in their carboxy domain, have been proposed to interact directly with purine-rich sequences $(5,6)$. Watakabe et al. (2) reported that a purine-rich sequence in exon M2 of the mouse IgM gene enhanced splicing of the upstream intron in an in vitro splicing system based on a heterologous intron of the Drosophila melanogaster doublesex (dsx) pre-mRNA that is spliced according to the nucleotide content of downstream exon sequence in the chimera. This model system could be used to analyze the splicing enhancer activity of the aforementioned purine-rich sequence, but has never been employed in studies of human diseases with splicing abnormalities.

As many as $15 \%$ of point mutations that result in human disease cause aberrant splicing (7). Recently, nonsense mutations located outside the splicing consensus sequence have been reported to cause skipping of the nonsense-containing exon in several human diseases (8-17). There are three possible mechanisms by which nonsense mutations could induce exon skipping: alteration of pre-mRNA secondary structures (10); a change in the mRNA coding potential (8); and disruption of the SES $(9,14)$. Although the second explanation, which was proposed to apply to Marfan syndrome, was confirmed in an in vitro study (18), there is no direct evidence that disruption of an SES can cause exon skipping in a genetic disease.

The severe disease Duchenne muscular dystrophy (DMD) and the more benign Becker muscular dystrophy (BMD) are allelic conditions characterized by progressive muscular degeneration and wasting caused by mutations in the dystrophin gene, which consists of 79 exons spread over $>3,000 \mathrm{~kb}$ of the $\mathrm{X}$ chromosome $(19,20)$. The dystrophin gene is characterized by an extremely low exon to intron ratio $(<0.01)$ and by the presence of multiple promoters and alternative splice sites (21). A purine-rich sequence within some exons of the complex dystrophin transcript is proposed to act as an additional cis-element to permit correct splice site selection. Several reports, all from our laboratory, of mutation-induced complete exon skipping in the dystrophin transcript that do not involve a

1. Abbreviations used in this paper: ASLV, avian sarcoma-leukosis virus; BMD, Becker muscular dystrophy; DMD, Duchenne muscular dystrophy; dsx, doublesex; SES, splicing enhancer sequence. 
nucleotide change in the splicing consensus sequences support the above suggestion (22-24).

DMD cannot be treated therapeutically, but we have proposed it might be transformed into milder BMD by correcting the frame-shifted into the in-frame translational reading frame by artificially inducing an alternative splicing reaction $(24,25)$. However, there is no evidence that such alternative splicing events can occur naturally because dystrophin mRNA has not been subjected to detailed analysis in BMD patients. Here, we report a nonsense mutation that would be expected to cause DMD in the dystrophin gene of a patient with BMD. We show that the nonsense mutation is eliminated from a small part of transcript by exon skipping. The results of in vitro splicing experiments support the idea that SES disruption is one mechanism by which a nonsense mutation induces the exon skipping. This discovery provides direct evidence that DMD can be converted to BMD by modulating splicing.

\section{Methods}

Case. A 31-yr-old Japanese male was diagnosed as BMD in his early twenties on the basis of clinical features. At the age of 31 , when cardiac symptoms of exercise fatigue and palpitation developed, cardiac examination disclosed dilated cardiomyopathy. At this time, the patient's serum CK activity was 3,251 IU/liter (control $<200$ IU/liter). Muscle biopsy samples obtained from the brachial biceps showed discontinuous patchy dystrophin staining when tested with three different antibodies recognizing the $\mathrm{NH}_{2}$-terminal, rod, or $\mathrm{COOH}$-terminal domains, respectively. The staining pattern was the same in all fibers, although there are marked differences in muscle fiber size (data not shown). Dystrophin immunostaining of a cardiac muscle biopsy showed the same staining pattern (data not shown), confirming the diagnosis of BMD.

The patient's elder brother also had a high serum CK value, and cardiac examination again disclosed findings compatible with dilated cardiomyopathy. Furthermore, he was diagnosed as BMD on the basis of the dystrophin staining pattern in a muscle biopsy. The patient's mother, maternal grandmother, and aunts also had histories of cardiac disease, but no further information was available.

Mutation analysis. Genomic DNA and cDNA were analyzed basically as described previously (26-30). Unless otherwise specified, all other PCR amplifications of genomic DNA or cDNA were performed according to the conditions described previously (22). To locate the mutation, a smaller fragment encompassing exons from 25 to 30 was amplified from muscle cDNA using a forward primer corresponding to a region in exon 25 (c25F; 5'-ACTCAGTGGGATCACATGTG-3') and a reverse primer complementary to a sequence in exon 30 (c30R; 5'-CTCCTGGGCAGACTGGATGC-3'). To quantify each transcript, cDNA synthesized from $1 \mu \mathrm{g}$ of total RNA was used for amplification and amplified products were quantified by measuring the density of each band (Densitograph; ATTO Co., Tokyo, Japan) after ethidium bromide staining.

Sequencing of the amplified DNA fragment from genomic DNA or cDNA was performed as described previously $(30,31)$.

Analysis of the genomic DNA sequence at the $3^{\prime}$ end of intron 26. Since the sequence of the region upstream of exon 27 is not available, the $5^{\prime}$ flanking region of exon 27 was cloned by nested PCR using the method described previously (32). A Sau3A1 cassette (Takara Shuzo Co., Kyoto, Japan) was ligated to Sau3A1-digested genomic DNA according to the protocol suggested by the supplier (Fig. $1 a$ ). Then, two PCR reactions were carried out using first cassette primer C1 (Takara Shuzo Co.) and primer G27R corresponding to the $3^{\prime}$ flanking region of exon 27 (5'-AACTATGACCATGTATTGAC-3'), and then cassette primer $\mathrm{C} 2$ (Takara Shuzo Co.) and primer E27R corresponding to a region in exon 27 (5'-TCAGTAAGGAGTTTCACTTTCGCTTCTTTT-3') (Fig. 1 a). After sequencing the $3^{\prime}$ end of intron 26, a new primer, G27F, corresponding to the $5^{\prime}$ flanking region of exon 27 (5'-TATGGAAGAGACTGGAGTTC-3') (Fig. 1 b) was designed and used together with primer G27R to amplify the genomic region encompassing exon 27.

Examination of splicing enhancer activity. The splicing enhancer activity of natural and artificial purine-rich sequences was examined in vitro by analyzing splicing of the heterologous intron of the Drosophila melanogaster doublesex (dsx) gene pre-mRNA in HeLa cell nuclear extracts as described before (3). In this in vitro system, splicing of chimeric dsx pre-mRNA is dependent on the downstream se- a

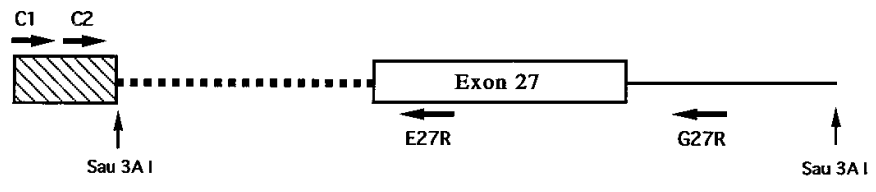

b

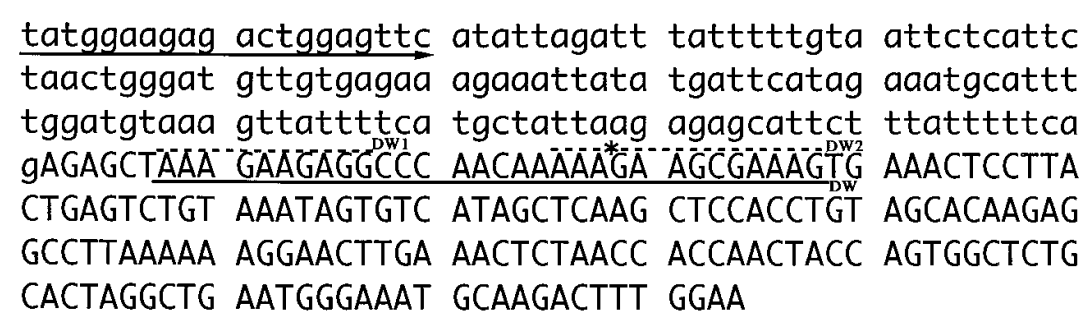

Figure 1. Genomic DNA sequence encompassing exon 27. (a) Schematic representation of the cloning of the $3^{\prime}$ end of intron 26 by nested PCR (32). The Sau3A1 cassette (Takara Shuzo Co.) and exon 27 are represented as shaded and open boxes, respectively. The dotted line represents the unknown sequence of intron 26. Horizontal arrows indicate the location and direction of primers used in the nested PCR. C1 and C2 primers were from Takara Shuzo Co. The vertical arrow shows the Sau3A1 restriction site. (b) Sequence of 3 ' end of intron 26. The nucleotide sequence encompassing the $3^{\prime}$ end of intron 26 and the complete exon 27 are shown (GenBank accession number AB116975). The 3' end of intron 26 includes a branching point, polypyrimidine tract, and conserved AG dinucleotide. Exon 27 has typical splice acceptor and weak splice donor sites (probability scores were 95.8 and 77.6 for splice acceptor and donor sites, respectively [43]). In the nucleotide sequence of the patient's DNA, nucleotide G3839 (44), corresponding to nucleotide 28 of exon 27, was replaced by a T (asterisk). Lowercase and uppercase letters indicate intron 26 and exon 27 sequences, respectively. The underlined region (DW) within exon 27 indicates the purine-rich region, which consists of two purine-rich sequences indicated by dotted lines over each (DW1 and DW2). The horizontal arrow indicates the location and direction of the G27F primer. 
Figure 2. Analysis of splicing enhancer activity. (a) Schematic representation of Drosophila melanogaster doublesex (dsx) chimeric plasmid. The chimeric plasmid consists of the entire third exon (168 nt), the third intron (114 nt), and $30 \mathrm{nt}$ of the $5^{\prime}$ portion of the fourth exon of the dsx gene and the linker sequence derived from the pSP72 vector (8 nt). The exons (boxes) and intron (line) are shown. The linker sequence (closed boxes) and the insertion site for the test sequence (open box) are indicated. $\mathrm{B}$ and $\mathrm{X}$ indicate BamHI and $\mathrm{XhoI}$ restriction enzyme recognition sites, respectively. The annealed test oligonucleotides were cloned into the insertion site. (b) Sequences examined for splicing enhancer activity. quence $(3,33)$. Briefly, template plasmids coding for the chimeric dsx pre-mRNA were constructed as shown in Fig. $2 a$. After confirming its sequence, radiolabeled pre-mRNA was prepared by in vitro transcription and subjected to in vitro splicing. The reaction products were electrophoresed and then quantified (BAS2000 Bio Image Analyzer; Fuji Photo Film Co., Tokyo, Japan) as described previously (24).

To evaluate splicing enhancer activity, various sequences were inserted between the BamH1 and Xho1 restriction enzyme recognition sites (Fig. $2 a$ ). Several test sequences were prepared (Fig. $2 b$ ) by annealing two strands of synthesized single-strand DNA. To minimize the effects of length variations, all prepared sequences were adjusted to $\sim 30$ nt. Pre-mRNA containing a segment of avian sarcoma-leukosis virus (ASLV) sequence that has been reported to be a good splicing enhancer (3) was used as positive control, and the BamHI-digested plasmid without any test sequences was used as a negative control.

\section{Results}

Once BMD had been diagnosed, the dystrophin gene was examined for the presence of a mutation. Deletion mutation analysis by multiplex PCR or Southern blot analysis failed to reveal any gross abnormality. Since RT nested PCR analysis of the dystrophin gene transcript in lymphocytes showed an aberrant splicing product (data not shown), the same region encompassing exons from 25 to 30 was amplified from muscle cDNA. Three products were obtained from the control (Fig. 3, lane $C$ ): the normal-sized product ( $717 \mathrm{bp}$ ) comprised the complete exon 25 to 30 sequence, a small ( $249 \mathrm{bp}$ ) product that lacked $468 \mathrm{nt}$ corresponding to exons 27, 28, and 29 (exon 27, $28,29^{-}$transcript), and an intermediate-sized product that was a heteroduplex of the normal and exon 27, 28, 29- transcripts (data reviewed at the first application). This showed that an exon $27,28,29^{-}$transcript is normally produced in skeletal muscle cells. This novel isoform is probably produced by alternative splicing specific to skeletal muscle.

Three different products were amplified from the patient's muscle cDNA (Fig. 3, lane $P$ ). One of these (534 bp) was specific to the patient's muscle cDNA, while the other two were the same as products amplified from control cDNA. Sequencing of the products disclosed that the largest one contained all exons but with a $\mathrm{G}$ to $\mathrm{T}$ transversion at nucleotide 28 of exon 27 , corresponding to nucleotide 3839 of dystrophin cDNA
(G3839T) (Fig. $4 a$ ). This mutation was expected to change a glutamic acid codon (GAA) to a nonsense codon (TAA). The patient-specific 534-bp product was completely devoid of exon 27 (183 bp exon; $27^{-}$transcript) (Fig. 4 b), resulting in the elimination of the nonsense mutation and the restoration of the translation reading frame. In fact, Western blot analysis of skeletal muscle disclosed the presence of a dystrophin polypeptide that was $\sim 27 \mathrm{kD}$ smaller and about five times less abundant than the 427-kD normal dystrophin (data not shown). Therefore, the nonsense mutation within exon 27 and the resulting exon $27^{-}$transcript were deemed to be the molecular explanation for the BMD phenotype of the index case.

The genomic DNA sequence around exon 27 was examined next, since exon skipping is usually induced by a mutation affecting the splicing consensus sequences. However, since the sequence of the region upstream of exon 27 was not available, this region was cloned from control DNA as described in

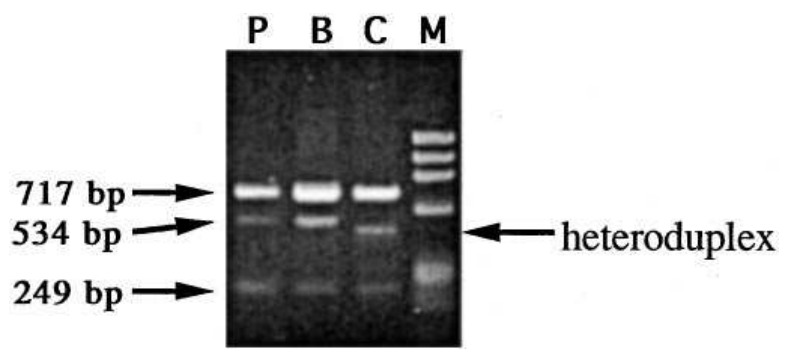

Figure 3. Amplification of dystrophin cDNA encompassing exons 25 to 30 . Amplified products encompassing exons 25 to 30 were obtained from muscle cDNA by RT-PCR. Two major products (717 and 249 bp) and other minor products were obtained from control (lane $C$ ). Three bands were visualized in the index case (lane $P$ ). Two of these (717 and $249 \mathrm{bp}$ ) corresponded to those from control cDNA, while the middle one (534 bp) was unique to the patient's muscle. The amplified product from muscle cDNA of the patient's elder brother showed the same pattern as lane $P$ (lane $B$ ). Lanes $P, B, C$, and $M$ represent the patient, his brother, control, and a size marker, respectively. The size marker was HaeIII-digested $\Phi$ X174 phage DNA (Takara Shuzo Co.). 


\section{G3839T}
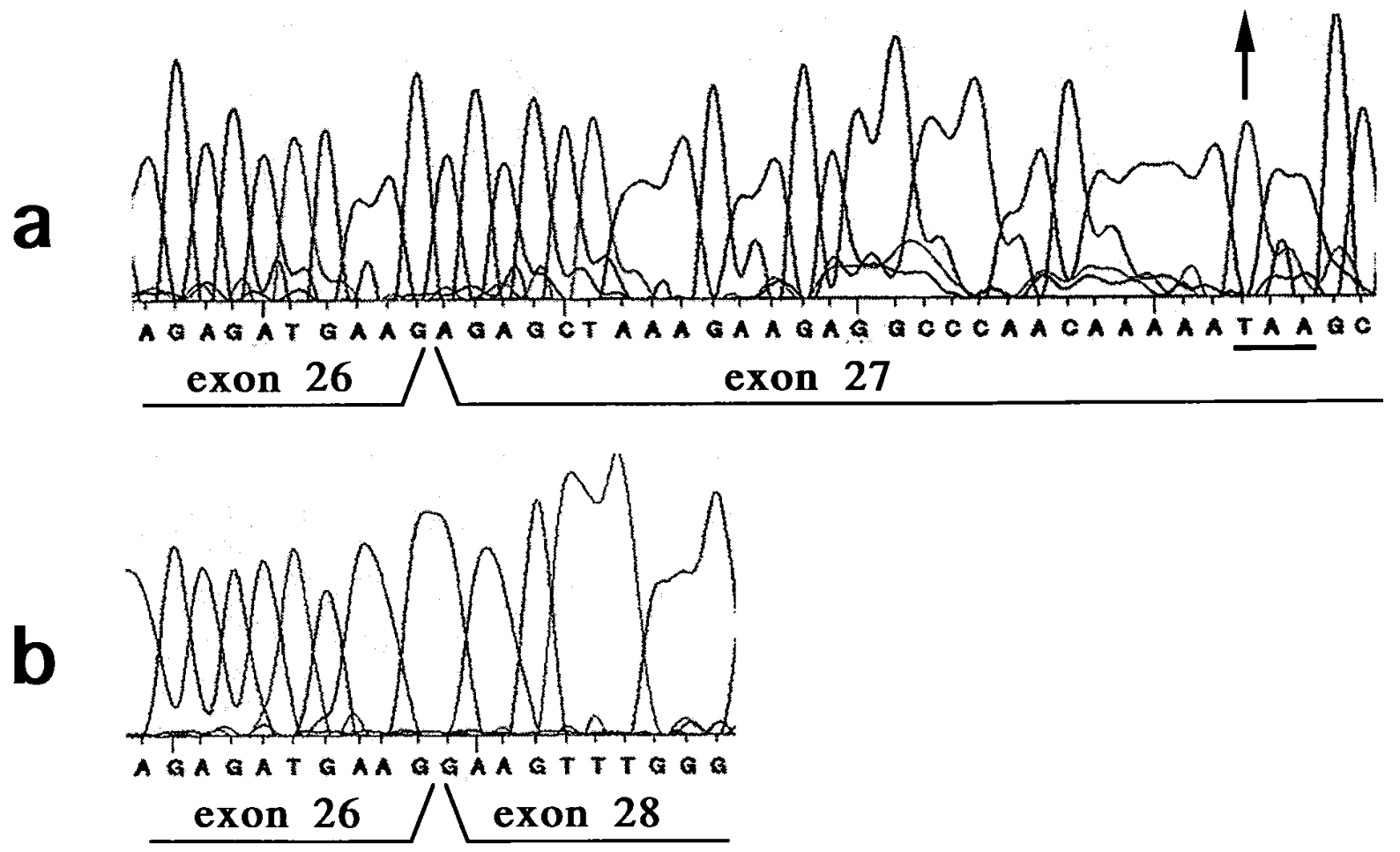

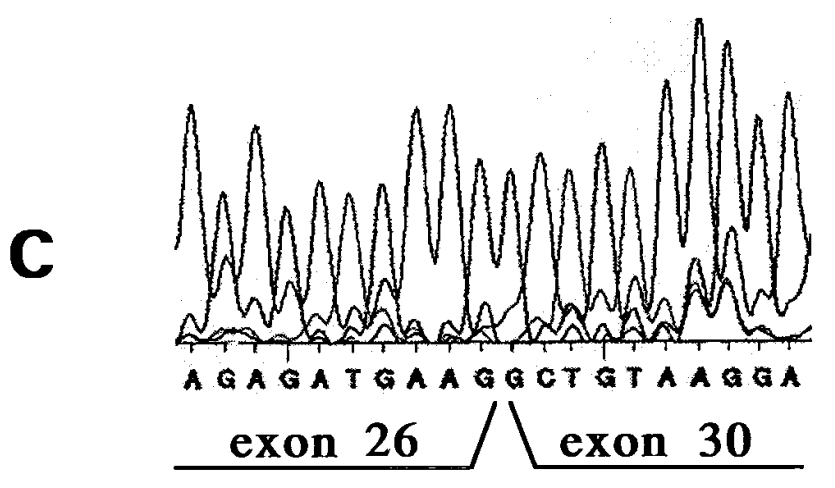

Methods (Fig. $1 a$ ) and a segment of $151 \mathrm{nt}$ from the $3^{\prime}$ end of intron 26 was determined (Fig. $1 b$ ). Analysis of the patient's DNA failed to disclose any nucleotide change at the branch point and 3' splice acceptor and 5' splice donor sites. However, the amplified DNA contained the same $G$ to $T$ nucleotide change at nucleotide 28 of exon 27 (data not shown). Since the same sequence was obtained from $>10$ independent amplified products, the possible existence of a mosaic sequence pattern could be excluded. In addition, analysis of genomic DNA and muscle cDNA from the patient's elder brother, who was also a BMD case, disclosed exactly the same splicing pattern (Fig. 3, lane $B$ ) and the G3839T nucleotide change (data not shown).

Although it has been proposed that nonsense codons decrease mRNA levels relative to wild-type mRNA because of increased instability or elimination by a nonsense codon recognition system (34), the majority of dystrophin mRNA in the index case contained the premature stop codon (Fig. 3, lane $P$ ). This indicated that the appearance of nonsense codon in exon
Figure 4. Sequence of the product amplified from the patient's skeletal muscle cDNA. Sequences of the regions joining exons 26 to 27, 28, or 30 are presented. (a) Normal splicing product. The sequence of the $3^{\prime}$ end of exon 26 (5'-TGAAG-3') is joined to the $5^{\prime}$ end of exon 27 (5'-AGAGC-3') in the largest fragment (top band of Fig. 3, lane $P$ ). Note the $\mathrm{G}$ to $\mathrm{T}$ transversion at nucleotide 28 of exon 27 (G3839T), creating the TAA nonsense codon (underline). (b) Exon 27-skipped product. The intermediate size product (middle band of Fig. 3, lane $P$ ) lacks all of exon 27 (nt 3812-3994) because the $3^{\prime}$ end of exon 26 is joined directly to the $5^{\prime}$ end of exon 28 (5'-GAAGT-3'). (c) Alternative splicing product. The sequence of the smallest band indicated that the $3^{\prime}$ end of exon 26 was joined directly to the $5^{\prime}$ end of exon 30 (5'-GCTGT-3'), indicating that it lacked exons 27, 28, and 29.

27 did not have a major impact on the levels of dystrophin transcript, and suggested that the nonsense codon did not affect the fate of this transcript.

The case reported here is the first example of dystrophinopathy in which exon skipping was actually induced by the nonsense mutation. To identify the factor responsible for inducing the observed exon skipping, we examined the region around exon 27 for cis-acting elements known to control splicing (Fig. $1 \mathrm{~b}$ ). Remarkably, a long purine-rich stretch, including two copies of the tandem AAG that is presumed to be a strong SES (3), was identified from nucleotide +7 to +31 of exon 27. This suggests that correct splicing of exon 27 needs this purine-rich region as a splicing enhancer $(2,5)$. Since the G3839T substitution is in the middle of this purine-rich region and affects the second tandem AAG (Fig. $1 b$ ), we reasoned that exon 27 skipping identified in the index case could be induced by disruption of SES.

The splice-enhancing activity of the purine-rich region of 


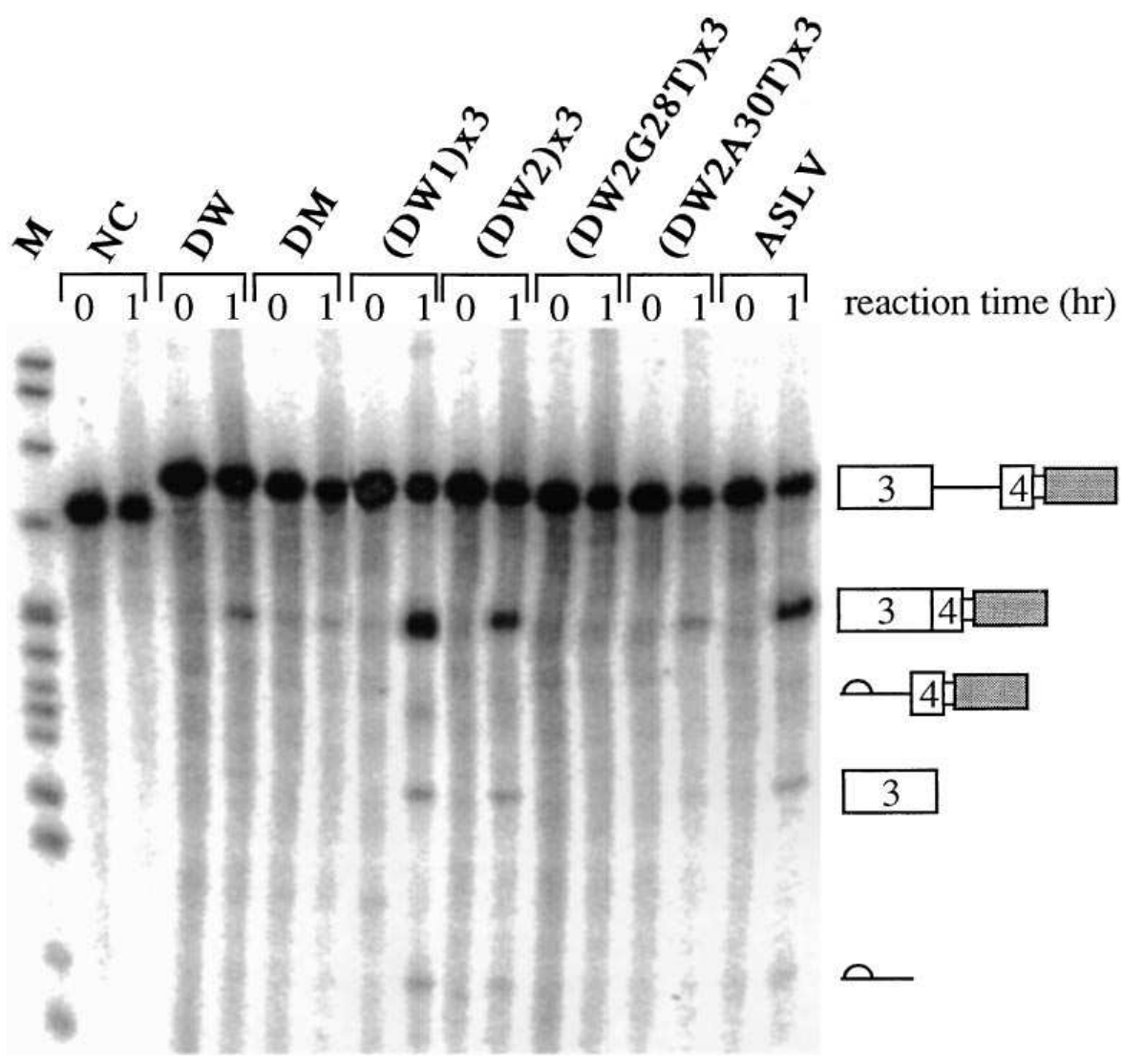

Figure 5. Analysis of splicing enhancer activity by in vitro splicing of chimeric dsx pre-mRNAs in a HeLa cell nuclear extract. The substrates used in this experiment contain the sequence listed in Fig. $2 b$ at the site indicated in Fig. $2 a$. The pre-mRNAs (10 fmol of each) were incubated in a HeLa cell nuclear extract at $30^{\circ} \mathrm{C}$ for the times indicated at the top of each lane, and then the product was applied to a $5 \%$ polyacrylamide gel containing $8 \mathrm{M}$ urea. The bands corresponding to the RNA products are shown schematically on the right side. The molecular size markers are fragments of ${ }^{32} \mathrm{P}$-labeled pBR322 obtained by MspI digestion (lane $M$ ). The splicing product of dsx pre-mRNA without an exon sequence was loaded in lane $N C$. The other lanes were loaded with splicing products obtained in reactions in which indicated sequence (Fig. $2 b$ ) was present in the insertion site. exon 27 was analyzed in vitro by its ability to activate splicing of chimeric dsx pre-mRNA, which is dependent on the downstream sequence (3). In this system, splicing of dsx pre-mRNA did not occur if any enhancer sequence was not present (negative control) (Fig. 5, lane $N C$ ), whereas splicing proceeded well when a part of the sequence of ASLV was inserted at the linker site (positive control) (Fig. 5, lane $A S L V$ ). When $31 \mathrm{nt}$ of the purine-rich sequence from $\mathrm{nt}+7$ to +37 of exon 27 (DW) was inserted into the linker site, the expected splicing product was observed (Fig. 5, lane $D W$ ). In contrast, the splicing product was not observed when the mutant sequence containing G3839T (DM) was inserted into the linker site (Fig. 5, lane $D M$ ). These in vitro studies clearly showed that the purine-rich sequence within exon 27 enhanced splicing of the upstream intron and that G3839T completely abolished this splicing enhancer activity.

The purine rich sequence from nt +7 to +37 of exon 27 comprises two purine-rich regions, $\mathrm{nt}+7$ to +16 [AAAGAAGAGG (DW1)] and $n t+25$ to +37 [AAAGAAGCGAAAG (DW2)] separated by three cytosines (Fig. $1 \mathrm{~b}$ ). To see which of the two purine-rich regions had stronger splicing enhancer activity, each part was examined individually. To avoid length effects and to facilitate manipulations, $\sim 30 \mathrm{nt}$ long sequences were prepared by synthesizing three tandem repeats of each part of the sequence (Fig. 2 b). Polypurines consisting of three repeats of the first part [(DW1)x3] and the second part [(DW2)x3] showed splicing enhancer activity, with the former exerting stronger splicing enhancer activity than the latter [Fig. 5 , lanes $(D W 1) \times 3$ and $(D W 2) \times 3]$. These data showed that the purine-rich sequence itself had the splicing enhancer activity but indicated that the activity depended on its nucleotide content or sequence. To determine the effect of the G3839T nucleotide change, the third G of DW2 was changed to T in each of the three tandem repeat sequences in (DW2)x3 to give (DW2G28T)x3. In this case, the splicing product was not observed [Fig. 5, lane (DW2G28T)x3]. Thus, the G to T substitution corresponding to G3839T suppressed splicing enhancer activity both in the original $31 \mathrm{nt}$ sequence of exon 27 and in the artificial purine-rich region.

There were two ways that G3839T might have suppressed splicing: the $\mathrm{T}$ insertion disrupted the purine-rich region (3) or a nonsense codon was created (8). Since the $\mathrm{G}$ to $\mathrm{T}$ change did indeed introduce a nonsense codon in the in vitro experiments, the effect of a simple $T$ nucleotide change that did not create a stop codon in any frame was examined by preparing another polypurine sequence that consisted of three AAAGATGCGCGAAAAG tandem repeats in which $A$ was replaced by $T$ at a position corresponding to nt 30 of exon 27 [(DW2A30T)x3] (Fig. 2 b). However, this sequence showed splicing enhancer activity [Fig. 5, lane (DW2A30T) x3] which was lower than that of the polypurine [(DW2)x3] but higher than that of the polypurine with a nonsense codon [(DM2G28T)x3]. These results showed that the $\mathrm{T}$ insertion itself inhibited SES function but that the inhibition was strengthened by the creation of nonsense codon.

Our data showed that a case of BMD resulted from partial elimination of nonsense mutation from dystrophin mRNA by exon skipping, and that the exon skipping observed in this 
BMD patient was due to disruption of an SES. This represents a novel case of BMD molecular pathogenesis that provides a natural model for how the severe DMD phenotype can be reduced to the less-severe BMD phenotype by altering the pattern of splicing.

\section{Discussion}

In this report, we describe in vitro evidence that disruption of the SES by a nonsense mutation is the cause of skipping of the mutation-containing exon. The mechanism of exon skipping induced by nonsense mutations has recently attracted considerable attention $(8,10-16)$. Three explanations have been proposed for the induction of exon skipping: a change in premRNA structure, a change in the mRNA coding potential, and disruption of SES. In support of the first of these explanations, alteration of pre-mRNA secondary structure by a nonsense mutation has been reported to lead to exon skipping in the Fancomi anemia group C gene (10), as did a missense mutation (35). This is supported by the recent finding that a silent mutation of the platelet fibrinogen receptor gene induced exon skipping (36). The nonsense mutation scanning model (the second explanation) is based on the observation that skipping of a constitutive fibrillin gene exon containing a nonsense mutation occurred even though the consensus splice sequence was maintained (8). In this model, the exon might be removed from mature mRNA if an open reading frame is not detected due to the presence of a premature termination codon. This model was confirmed by an in vitro model splicing system (18). Furthermore, exon skipping has been reported to be due to the creation of a stop codon in several diseases $(8,11,12,16)$. However, this model is not universally accepted because nonsense mutations do not necessarily induce splicing alterations (37).

The third explanation for the induction of exon-skipping reactions by nonsense mutations, which invokes disruption of splicing enhancers, is based on the finding that a purine-rich sequence activates the splicing of upstream introns $(2,3,38)$. This theory was supported by Santisteban et al. who reported that exon skipping was induced by a $\mathrm{C}$ to $\mathrm{T}$ change in the purine-rich region of exon 5 of the adenosine deaminase gene (14). Furthermore, disruption of an SES was thought to cause the skipping of the nonsense-containing exon resulting from mutations in the purine-rich sequence of the Factor VIII gene (9) and the cystic fibrosis transmembrane conductance regulator gene $(13,15)$. However, these examples were not confirmed by in vitro experiments. In addition to the above, the nonsense-containing exon has been reported to be eliminated spontaneously by alternative splicing, a mechanism proposed to explain the phenotypic rescue of a common inherited defect (39).

In our case, the secondary structure alteration theory is not plausible because the mutation was not predicted to cause a marked change in the secondary structure of the pre-mRNA region encompassing exon 27 (data not shown). The nonsense mutation scanning model also seemed not to apply because the transcript containing the nonsense codon remained a major component of the total transcript (Fig. 3, lane $P$ ). Finally, we focused on the idea that disruption of the SES could cause missplicing. The in vitro splicing studies showed that the wild-type purine-rich sequence of exon 27 had splicing enhancer activity and that this activity was completely abolished by the G3839T substitution (Fig. 5, lane DM). However, induction of exon 27 skipping was observed only in a small proportion of the dystrophin transcripts in skeletal muscle (Fig. 3, lane $P$ ), even though the results of the in vitro splicing analysis showed that the mutant sequence completely abolished splicing enhancer activity (Fig. 5, lane DM). Since exon 27 has two separate purine-rich regions, each of which showed splicing enhancer activity [Fig. 5 , lanes ( $D W 1) \times 3$ and $(D W 2) \times 3$ ], it seemed likely that disruption of the second part of the purine-rich region by the $\mathrm{G}$ to $\mathrm{T}$ substitution might not be enough to abolish SES function in vivo. In fact, synergistic interactions of two splicing enhancers within an exon have been reported (33). Therefore, normal splicing presumably proceeded in this case even when one of two purine-rich regions was disrupted. Recently, members of the SR family of essential splicing factors have been proposed to interact directly with purine-rich sequences $(5,6)$, raising the possibility that SESs might function through their ability to recruit one or more SR proteins. Our observation that exon 27 skipping was incomplete in skeletal muscle suggested that nuclear proteins present in HeLa cell nuclear extracts were different from those present in skeletal muscle cells. Further investigation is required to clarify the exact interaction of each purine-rich region with nuclear proteins.

The in vitro splicing study showed that a sequence containing a nonsense codon inhibited splicing of an upstream intron more efficiently than a similar mutation that did not contain a stop codon [Fig. 5, lanes (DW2G28T) x3 and (DW2 A30T)x3]. This evidence supported the idea that creation of a nonsense codon contributed to aberrant splicing (8) but it is not clear whether this mechanism contributed to the situation observed in the patient's muscle because of the large amounts of nonsense codon-containing transcripts that were present. Thus, the in vitro splicing study did not faithfully reproduce the in vivo phenomenon and it remains possible that the nonsense codon recognition model could explain a small part of the induced exon skipping in this case. A survey of similar nonsense mutations in purine-rich regions that induce exon skipping $(9,13-15)$ revealed that they all introduced a $\mathrm{T}$ nucleotide, which conforms to the finding that thymine has a strong propensity for SES disruption (3). Therefore, exon skipping should be evaluated whenever a stop codon created by $\mathrm{T}$ replacement is identified in purine-rich region of a disease-causing gene.

This is the fourth report of a nonsense mutation of the dystrophin gene that caused BMD rather that DMD (40). These examples of an apparent lack of correlation between the presence of a nonsense mutation and the severity of the disease were explained as follows: one nonsense mutation was found to be a somatic mosaic (41), while the two others disappeared spontaneously from mRNA because they were located in exons that normally undergo alternative splicing (42). In the index case, partial skipping of exon 27 removed a nonsense mutation from some of the dystrophin mRNA and resulted in the production of truncated dystrophin. Among 79 exons of the dystrophin gene, only a limited number have a purine-rich region that might be an SES. Therefore, rescuing of nonsense mutation by exon skipping is likely to be a very rare occurrence in dystrophinopathy. Nevertheless, our finding has very important clinical implications for the proposed therapeutic conversion of DMD into BMD by artificially inducing a reading frame change through altered splicing using the antisense nucleotide strategy (25). 


\section{Acknowledgments}

We thank Dr. Anthony Pugsley for advice and for critically reading the manuscript, and Dr. Yoshihiro Ishida for help with obtaining samples and clinical information from family members. We acknowledge Ms A. Maruyama for secretarial help.

This work was supported by grants from the Ministry of Education, Science and Culture of Japan, the National Center of Neurology and Psychiatry (NCNP) of the Ministry of Health and Welfare of Japan, and Hyogo Sozo Kyokai.

\section{References}

1. Green, M.R. 1986. Pre-mRNA splicing. Annu. Rev. Genet. 20:671-708.

2. Watakabe, A., K. Tanaka, and Y. Shimura. 1993. The role of exon sequences in splice site selection. Genes Dev. 7:407-418.

3. Tanaka, K., A. Watakabe, and Y. Shimura. 1994. Polypurine sequences within a downstream exon function as a splicing enhancer. Mol. Cell. Biol. 14: 1347-1354.

4. Tian, M., and T. Maniatis. 1994. A splicing enhancer exhibits both constitutive and regulated activities. Genes Dev. 8:1703-1712.

5. Yeakley, J.M., J.-P. Morfin, M.G. Rosenfeld, and X.-D. Fu. 1996. A complex of nuclear proteins mediates SR protein binding to a purine-rich splicing enhancer. Proc. Natl. Acad. Sci. USA. 96:7582-7587.

6. Tacke, R., and J.L. Manley. 1995. The human splicing factors ASF/SF2 and SC35 possess distinct, functionally significant RNA binding specificities. EMBO (Eur. Mol. Biol. Organ.) J. 14:3540-3551.

7. Krawczak, M., J. Reiss, and D.N. Cooper. 1992. The mutational spectrum of single base-pair substitutions in mRNA splice junctions of human genes: causes and consequences. Hum. Genet. 90:41-54.

8. Dietz, H.C., D. Valle, C.A. Francomano, R.J. Kendzior, R.E. Pyeritz, and G.R. Cutting. 1993. The skipping of constitutive exons in vivo induced by nonsense mutations. Science (Wash. DC). 259:680-683.

9. Naylor, J.A., P.M. Green, C.R. Rizza, and F. Giannelli. 1992. Analysis of factor VIII mRNA reveals defects in every one of 28 haemophilia A patients. Hum. Mol. Genet. 2:11-17.

10. Gibson, R., A. Hajiapour, M. Murer-Orland, M. Buchwald, and C. Mathew. 1993. A nonsense mutation and exon skipping in the Fancomi anaemia group C gene. Hum. Mol. Genet. 2:797-799.

11. Bach, G., S.M. Moskowitz, P.T. Tieu, A. Matynia, and E.F. Neufeld. 1993. Molecular analysis of hurler syndrome in Druze and Muslim Arab patients in Israel: multiple allelic mutations of the IDUA gene in a small geographic area. Am. J. Hum. Genet. 53:330-338.

12. Das, S., B. Levinson, S. Whieney, C. Vulpe, S. Packman, and J. Gitschier. 1994. Diverse mutations in patients with Menkes disease often lead to exon skipping. Am. J. Hum. Genet. 55:883-889.

13. Hull, J., S. Shackleton, and A. Harris. 1994. The stop mutation R553X in the CFTR gene results in exon skipping. Genomics. 19:362-364.

14. Santisteban, I., F.X. Arredondo-Vega, S. Kelly, M. Loubser, N. Meydan, C. Roifman, P.L. Howell, T. Bowen, K.I. Weinberg, M.L. Schroeder, and M. Hershfield. 1995. Three new adenosine deaminase mutations that define a splicing enhancer and cause severe and partial phenotypes: implications for evolution of a CpG hotspot and expression of a transduced ADA cDNA. Hum. Mol. Genet. 4:2081-2087.

15. Will, K., T. Dork, M. Stuhrmann, H. von der Hardt, H. Ellemunter, B. Tummler, and J. Schmidthe. 1995. Transcript analysis of CFTR nonsense mutations in lymphocytes and nasal epithelial cells from cystic fibrosis patients. Hum. Mutat. 5:210-220.

16. Okamoto, Y., T. Yamazaki, A. Katsumi, T. Kojima, J. Takamatsu, M. Nishida, and H. Saito. 1996. A novel nonsense mutation associated with an exon skipping in a patient with hereditary protein S deficiency type 1. Thromb. Haemostasis. 75:877-882.

17. Bienvenu, T., C. Beldjord, J. Chelly, N. Fonknechten, D. Hubert, D. Dusser, and J.C. Kaplan. 1996. Analysis of alternative splicing patterns in the cystic fibrosis transmembrane conductance regulator gene using mRNA derived from lymphoblastoid cells of cystic fibrosis patients. Eur. J. Hum. Genet. 4:127-134.

18. Dietz, H.C., and R.J. Kendzior. 1994. Maintenance of an open reading frame as an additional level of scrutiny during splice site selection. Nat. Genet. 8:183-188.

19. Ahn, A.H., and L.M. Kunkel. 1993. The structural and functional diversity of dystrophin. Nat. Genet. 3:283-291.

20. Nishio, H., Y. Takeshima, N. Narita, H. Yanagawa, Y. Suzuki, Y. Ishikawa, Y. Ishikawa, R. Minami, H. Nakamura, and M. Matsuo. 1994. Identification of a novel first exon in the human dystrophin gene and of a new promoter located more than $500 \mathrm{~kb}$ upstream of the nearest known promoter. J. Clin. Invest. 94:1037-1042.
21. Matsuo, M. 1996. Duchenne/Becker muscular dystrophy: from molecular diagnosis to gene therapy. Brain Dev. 18:167-172.

22. Matsuo, M., T. Masumura, H. Nishio, T. Nakajima, Y. Kitoh, T. Takumi, J. Koga, and H. Nakamura. 1991. Exon skipping during splicing of dystrophin mRNA precursor due to an intraexon deletion in the dystrophin gene of Duchenne muscular dystrophy Kobe. J. Clin. Invest. 87:2127-2131.

23. Narita, N., H. Nishio, Y. Kitoh, Y. Ishikawa, Y. Ishikawa, R. Minami, H. Nakamura, and M. Matsuo. 1993. Insertion of a 5' truncated L1 element into the $3^{\prime}$ end of exon 44 of the dystrophin gene resulted in skipping of the exon during splicing in a case of Duchenne muscular dystrophy. J. Clin. Invest. 91: 1862-1867.

24. Takeshima, Y., H. Nishio, H. Sakamoto, H. Nakamura, and M. Matsuo. 1995. Modulation of in vitro splicing of the upstream intron by modifying an intraexon sequence which is deleted from the dystrophin gene in dystrophin Kobe. J. Clin. Invest. 95:515-520.

25. Pramono, Z.A.D., Y. Takeshima, H. Alimsardjono, A. Ishii, S. Takeda, and M. Matsuo. 1996. Induction of exon skipping of the dystrophin transcript in lymphoblastoid cells by transfecting an antisense oligodeoxynucleotide complementary to an exon recognition sequence. Biochem. Biophys. Res. Commun. 226:445-449.

26. Chamberlain, J.S., R. Gibbs, J. Ranier, and C. Caskey. 1990. Multiplex PCR for the Diagnosis of Duchenne Muscular Dystrophy. Academic Press, San Diego. 272-281.

27. Beggs, A.H., M. Koenig, F.M. Boyce, and L.M. Kunkel. 1990. Detection of $98 \%$ of $\mathrm{DMD} / \mathrm{BMD}$ gene deletions by polymerase chain reaction. Hum. Genet. 86:45-48.

28. Boyce, F.M., A.H. Beggs, C. Feener, and L.M. Kunkel. 1991. Dystrophin is transcribed in brain from a distant upstream promoter. Proc. Natl. Acad. Sci. USA. 88:1276-1280.

29. Roberts, R.G., M. Bobrow, and D.R. Bentley. 1992. Point mutations in the dystrophin gene. Proc. Natl. Acad. Sci. USA. 89:2331-2335.

30. Hagiwara, Y., H. Nishio, Y. Kitoh, Y. Takeshima, N. Narita, H. Wada, M. Yokoyama, H. Nakamura, and M. Matsuo. 1994. A novel point mutation $\left(\mathrm{G}^{-1}\right.$ to $\left.\mathrm{T}\right)$ in a $5^{\prime}$ splice donor site of intron 13 of the dystrophin gene results in exon skipping and is responsible for Becker muscular dystrophy. Am. J. Hum. Genet. 54:53-61.

31. Pokharel, R.K., H. Alimsardjono, M. Matsuo, T. Takashima, and R. Shiba. 1997. Highly recurrent G1138A mutation of fibroblast growth factor receptor 3 (FGFR3) gene identified in achondroplasia, the commonest form of chondrodysplasia. J. Nep. Med. Assoc. 35:52-57.

32. Isegawa, Y., J. Sheng, Y. Sokawa, K. Yamanishi, O. Nakagomi, and S. Ueda. 1992. Selective amplification of cDNA sequence from total RNA by cassette-ligation mediated polymerase chain reaction (PCR): application to sequencing 6-5 kb genome segment of hantavirus strain B-1. Mol. Cell. Probes. 6: $467-475$.

33. Lynch, K.W., and T. Maniatis. 1995. Synergistic interactions between two distinct elements of a regulated splicing enhancer. Genes Dev. 9:284-293.

34. Maquat, L.E. 1995. When cells stop making sense: effects of nonsense codons on RNA metabolism in vertebrate cells. RNA. 1:453-465.

35. Steingrimsdottir, H., G. Rowley, G. Dorado, J. Cole, and A.R. Lehmann. 1992. Mutations which alter splicing in the human hypoxanthine-guanine phosphoribosyltransferase. Nucl. Acids Res. 20:1201-1208.

36. Jin, Y., H.C. Dietz, R.A. Montgomery, W.R. Bell, I. McIntosh, B. Coller, and P.F. Bray. 1996. Glanzmann thrombasthenia. J. Clin. Invest. 98:17451754 .

37. Willing, M.C., S.P. Deschenes, R.L. Slayton, and E.J. Roberts. 1996. Premature chain termination is a unifying mechanism for COL1A1 null alleles in osteogenesis imperfecta type I cell strains. Am. J. Hum. Genet. 59:799-809.

38. Xu, R., J. Teng, and T.A. Cooper. 1993. The cardiac troponin T alternative exon contains a novel purine-rich positive splicing element. Mol. Cell. Biol. 13:3660-3674.

39. Morisaki, H., T. Morisaki, L.K. Newby, and E.W. Holmes. 1993. Alternative splicing: a mechanism for phenotypic rescue of a common inherited defect. J. Clin. Invest. 91:2275-2280.

40. Monaco, A.P., C. Bertelson, S. Liechti-Gallati, H. Moser, and L. Kunkel. 1988. An explanation for the phenotypic differences between patients bearing partial deletions of the DMD locus. Genomics. 2:90-95.

41. Prior, T.W., C. Bartolo, A.C. Papp, P.J. Snyder, M.S. Sedra, A.H.M. Burghes, and J.R. Mendell. 1996. Nonsense mutations in a Becker muscular dystrophy and an intermediate patient. Hum. Mutat. 7:72-75.

42. Barbieri, A.M., N. Soriani, A. Ferlini, A. Michelato, M. Ferrari, and P. Carrera. 1996. Seven novel additional small mutations and a new alternative splicing in the human dystrophin gene detected by heteroduplex analysis and restricted RT-PCR heteroduplex analysis of illegitimate transcripts. Eur. J. Hum. Genet. 4:183-187.

43. Shapiro, M.B., and P. Senapathy. 1987. RNA splice junctions of different classes of eukaryotes: sequence statics and functional implications in gene expression. Nucl. Acids Res. 15:7155-7174.

44. Koenig, M., A.P. Monaco, and L.M. Kunkel. 1988. The complete sequence of dystrophin predicts a rod-shaped cytoskeletal protein. Cell. 53:219228. 